\title{
Cost is not a barrier to implementing clopidogrel pharmacogenetics
}

Larisa H. Cavallari, Pharm.D., and Glen T. Schumock, Pharm.D., M.B.A.

From the Department of Pharmacy Practice (both authors), and the Center for

Pharmacoeconomic Research (Dr. Schumock), College of Pharmacy, University of Illinois at Chicago, 833 South Wood St, Rm 164, Chicago, IL

Author for correspondence and reprint requests: Larisa H. Cavallari, Department of Pharmacy Practice, University of Illinois at Chicago College of Pharmacy, 833 S. Wood St, Room 164, Chicago, IL 60612-7230; Tel: (312) 996-0886; Fax: (312) 996-0379; Email: humma@uic.edu 


\section{The evidence gap}

Clopidogrel in combination with aspirin is widely prescribed to prevent cardiovascular events in patients with an acute coronary syndrome (ACS) or percutaneous coronary intervention (PCI). Clopidogrel requires biotransformation to its active thiol metabolite, which irreversibly binds to the platelet $\mathrm{P}_{2} \mathrm{Y}_{12}$ adenosine diphosphate receptor to inhibit platelet activation. Cytochrome P450 (CYP) 2C19 is involved in both steps of the biotransformation process. Deficient CYP2C19 activity secondary to genetic polymorphism results in lower active metabolite levels and reduced inhibition of platelet aggregation with clopidogrel. ${ }^{1-3}$

The CYP2C19*1/*1 genotype is the wild-type genotype associated with normal enzyme activity and the extensive metabolizer (EM) phenotype. The CYP2C19*2 (c.681G>A) allele is the most common variant leading to loss of CYP2C19 function, with carriers of one $\left({ }^{*} 1 / * 2\right)$ or two $(* 2 / 2)$ CYP2C19*2 alleles deemed intermediate metabolizers (IMs) or poor metabolizers (PMs), respectively. On the other hand, the CYP2C19*17 allele confers increased enzyme activity, higher levels of the active clopidogrel metabolite, and the ultra-rapid metabolizer (UM) phenotype. $^{4}$

Numerous studies have documented an increased risk for cardiovascular events following ACS and PCI in clopidogrel-treated patients with the CYP2C19 PM or IM phenotype compared

to similarly treated EMs or UMs. ${ }^{2,3,5-7}$ Some of the most convincing data come from the Trial to Assess Improvement in Therapeutic Outcomes by Optimizing Platelet Inhibition with Prasugrel Thrombolysis in Myocardial Infarction (TRITON-TIMI) 38 trial, in which patients with ACS and planned PCI where randomized to clopidogrel or prasugrel. ${ }^{8}$ Clopidogrel-treated participants with a loss-of-function CYP2C19 allele (PM or IM phenotype) had a significantly higher risk for the composite endpoint of death from cardiovascular causes, myocardial 
infarction, or stroke and a 3-fold increase in the risk of stent thrombosis compared to noncarriers. ${ }^{3}$

In 2010, the clopidogrel labeling was revised to include a boxed warning about reduced drug effectiveness in CYP2C19 PMs. ${ }^{9}$ The labeling advises healthcare professionals that genotyping is available and to consider alternative strategies in patients found to be PMs. Prasugrel and ticagrelor are third generation $\mathrm{P}_{2} \mathrm{Y}_{12}$ receptor inhibitors that have a more rapid onset of action and are superior in preventing cardiovascular events compared to clopidogrel, but at the cost of increased non-coronary after bypass graft-related bleeding. ${ }^{8,10-12}$ Neither agent is affected by CYP2C19 loss-of-function genotype, and thus, either may be considered as an alternative to clopidogrel in individuals with a CYP2C19 loss-of-function allele. ${ }^{13,14}$ However, prasugrel is contraindicated in patients with a history of transient ischemic attack or stroke, and ticagrelor is more expensive than clopidogrel and requires twice daily dosing, somewhat limiting use of these agents. ${ }^{15}$ In addition, clopidogrel is expected to be available in generic form in 2012, which could significantly widen the cost differential among antiplatelet agents.

For both practitioners and payers, it may be unclear exactly how to balance the differences in clinical effectiveness and safety together with cost implications when choosing one antiplatelet drug versus another for patients with an ACS or PCI. Using genotyping to guide selection of antiplatelet therapy has been suggested as one means of sorting through these prescribing complexities. ${ }^{16}$ However, to date this has not been widely implemented in clinical practice, despite both the aforementioned data supporting reduced clopidogrel effectiveness in CYP2C19 PMs and IMs and the revised clopidogrel labeling. An obstacle to clinical implementation of pharmacogenomics is the need to establish clinical utility and cost effectiveness of a genotype-guided therapeutic approach. Trials addressing the clinical utility of 
genotype-guided antiplatelet therapy are underway. The data presented by Reese et $\mathrm{al}^{17}$ in this issue of Pharmacotherapy provide evidence of the cost-effectiveness of such an approach.

\section{New findings}

The authors conducted a model-based cost-effectiveness study comparing genotype guided therapy (arm 1), to either clopidogrel for all patients (arm 2) or prasugrel for all patients

(arm 3), regardless of genotype. ${ }^{17}$ Patients included in the model were assumed to have ACS and scheduled PCI. The decision model incorporated the probability that a patient in the genotypeguided therapy arm would have a loss-of-function allele (i.e., IM or PM phenotype), in which case, the patient was assumed to receive prasugrel, whereas those deemed EMs or UMs were assumed to receive clopidogrel.

All three arms of the model incorporated the probability that patients would experience a cardiovascular event (cardiovascular death, non-fatal myocardial infarction, or non-fatal stroke), a non cardiovascular event (major or minor bleeding), or no event, while receiving antiplatelet therapy during the 15-month post-PCI period. The probabilities for these events were obtained from the TRITON-TIMI 38 trial, in which prasugrel was shown to be superior to clopidogrel in preventing adverse cardiovascular events in ACS patients with planned PCI. ${ }^{8}$ The probability of the genotype result (i.e. genotype frequencies) was obtained from the TRITON-TIMI 38 genetic sub-study. ${ }^{13}$ Also included in the model were 1) the cost of hospitalization and PCI, 2) the cost of genotyping (only in the genotype arm), 3) the cost of antiplatelet therapy (for 15 months), and 4) the cost associated with each CV or non-CV event. The perspective was that of the third party payer, and cost-effectiveness was expressed as the incremental cost per event avoided in the 
genotype-guided therapy arm versus clopidogrel or prasugrel for all patients. This is reported as the incremental cost-effectiveness ratio or ICER.

The investigators performed the analysis by simulating patients in each arm of the model (10,000 patients per arm) to determine the number of CV events avoided and then the number of patients who would need to be genotyped in order to prevent the occurrence of one cardiovascular event in the other arms (e.g., number needed to treat, NNT). Keeping with contemporary practices in decision modeling, probabilistic sensitivity analysis was used to test the robustness of the model results over a range of probability estimates from the literature. Cost-effectiveness acceptability curves (CEACs) were then created using Monte Carlo simulations to present the probability that genotype-guided therapy is cost-effective compared to either clopidogrel or prasugrel for all patients over a range of "willingness-to-pay” (WTP) values, which is explained further below.

The authors found that genotype-guided antiplatelet therapy was more cost effective than either clopidogrel or prasugrel treatment in all patients. That is, when one considers the cost of genotyping along with the cost of antiplatelet drug, and other costs associated with treatment (including costs associated with cardiovascular events and bleeding), the genotyped-guided approach is both less expensive and more effective. The ICER for the comparison of genotypeguided antiplatelet therapy to clopidogrel was $\$-6,760$ and the ICER for genotyping compared to prasugrel was \$ -11,710. In pharmacoeconomic terms, when one option is less expensive and more effective, it is considered "dominant" compared to the alternative. The cost-effectiveness of genotype-guided therapy was largely driven by the fact that, among 10,000 patients, this strategy produced 450 fewer cardiovascular events compared to clopidogrel treatment for all and 350 fewer events compared to prasugrel treatment for all. Thus the NNT with genotype-guided 
therapy (or essentially the number needed to genotype) to avoid one event compared to clopidogrel was 23 and compared to prasugrel was 30.

The probabilistic sensitivity analyses conducted by the investigators showed the result to be consistent over a wide range of values. This analysis produced CEACs expressed as the WTP (or cost incurred by the payer) to avoid one event. Essentially, the CEACs showed the proportion of time that genotype-guided therapy is cost-effective compared to either clopidogrel or prasugrel treatment for all at each level of WTP, with the "threshold" being defined as the WTP value where the payer could be 95\% certain to avoid one event. For the comparison to clopidogrel the threshold was $\$ 9,670$, and for prasugrel it was $\$ 22,500$. The WTP threshold can be interpreted as the cost per CV event avoided above which the third-party decision maker would choose to select the alternative (e.g., clopidogrel or prasugrel) instead of the genotypeguided therapy. These are important values from the standpoint of the third-party payer (e.g., insurance company) who may be in the position to make policy decisions for large groups of patients. The values suggest that the decision to use genotype-guided therapy is not likely to change unless there are large changes in the costs incorporated in the model.

An example of such a change in costs might be the introduction of generic clopidogrel. In order to anticipate this, the authors conducted a sensitivity analysis where the brand cost for clopidogrel was substituted with an assumed generic cost of $\$ 1$ per day. They then repeated all of the analyses presented above. Under this scenario, genotype-guided therapy was less costeffective than generic clopidogrel (though it remained so compared to prasugrel). In fact, the generic clopidogrel for all option dominated the genotype-guided therapy (ICER \$ 2,300). The WTP threshold for this analysis was $\$ 3,700$, meaning that the decision to select generic clopidogrel for all instead of genotype-guided therapy would not change until the cost of per 
event avoided exceeded $\$ 3,700$. It should be noted that this threshold is relatively low and thus minor changes in the costs or other assumptions used in the analysis might result in a different conclusion.

\section{Unanswered questions}

The authors should be congratulated for conducting one of the first studies to examine the cost-effectiveness of genotype-guided antiplatelet therapy. While their findings support such an approach, some clinical and economic questions remain and require consideration. First, the authors assumed event rates similar to those in TRITON-TIMI 38, in which the majority of patients (94\%) had an ACS and underwent intracoronary stenting (e.g., high risk). While the authors examined the most relevant population in terms of cardiovascular risk, their results may not be generalizable to lower risk patients because event probabilities will be different.

A related issue is that while a majority of patients taking antiplatelet agents are over age 65 years, the TRITON-TIMI 38 study (on which this model was based) included younger patients (median patient age of 61 years). Older patients may have different risks for cardiovascular and non-cardiovascular events than were used in this study. Therefore these results may not hold for that population.

Further, over 90\% of TRITON-TIMI 38 participants were Caucasian. Approximately 25\% to $30 \%$ of Caucasians carry a CYP2C19*2 allele, and 2\% to 4\% are PMs. Slightly more African Americans (35\%) and about twice as many Asians (60\%) carry a loss-of-function allele. ${ }^{18}$ The probabilistic sensitivity analysis adjusted for variation in phenotype frequency. However, the efficacy of prasugrel versus clopidogrel in non-Caucasian patients cannot be 
determined from the TRITON-TIMI 38 data, and thus the cost-effectiveness of genotype-guided therapy in non-Caucasians is unclear.

While the investigators focused solely on the CYP2C19 genotype, other genotypes have been implicated in clopidogrel responsiveness, mostly notable $A B C B 13435 \mathrm{C}>\mathrm{T} .{ }^{19,20}$ Additional genotyping may incur additional costs; however, it may also improve the ability to predict clopidogrel responsiveness. Cayla et $\mathrm{al}^{19}$ recently demonstrated that, among clopidogrel-treated patients, a combined model of clinical and genetic factors (including CYP2C19 and ABCB1 genotypes) was more predictive of risk for early stent thrombosis compared to clinical-only and genotype-only models. Use of such multi-factorial models may allow for refinement of risk with clopidogrel and further improve the cost effectiveness of a pharmacogenetic approach to antiplatelet therapy.

Other important clinical questions are when and who to genotype. The question of when to genotype is particularly relevant for patients with ACS or undergoing PCI, in whom antiplatelet loading is warranted. Patients with a loss-of-function CYP2C19 genotype do not obtain sufficient concentrations of the active clopidogrel metabolite with a standard loading dose, and presumably, would derive greater protection against acute coronary events with an alternative antiplatelet strategy. ${ }^{21}$ Rapid or point-of-care genotyping would be required to identify these patients prior to PCI. In the absence of such, one approach would be to load all patients on prasugrel or ticagrelor. Then, based on evidence that clopidogrel and prasugrel provide similar protection against cardiovascular events in the absence of a CYP2C19 loss-offunction genotype, patients later genotyped and found to be EMs or UMs could be transitioned to clopidogrel. $^{22}$ This approach would provide rapid and effective platelet inhibition acutely, and then allow for use of the most cost-effective chronic therapy. ${ }^{10}$ As far as who to genotype, the 
NIH-supported Clinical Pharmacogenetics Implementation Consortium (CPIC) suggest one of two approaches: 1) genotype all patients who suffer an ACS or undergo PCI or 2) genotype only moderate to high-risk patients, including those with a history of stent thrombosis, diabetes, renal insufficiency, or high risk coronary angiographic features. ${ }^{23}$ Consistent with the approach outlined above, standard dose clopidogrel is recommended in patients without a CYP2C19 lossof-function allele, while substitution of prasugrel or ticagrelor is a recommended strategy for IMs or PMs.

There are also economic questions that remain unanswered. While the study conducted several types of sensitivity analysis, including presentation of CEACs, some basic questions that practitioners and payers are likely to wonder remain unaddressed. For example, while the study provides results for a scenario of generic clopidogrel at $\$ 1 /$ day, the actual price of generic clopidogrel may be different. Therefore it would be better to know the clopidogrel price threshold where a different decision would be made (i.e., how expensive does generic clopidogrel have to be for genotype-guided therapy to be the preferred option?) The same is true for the price of prasugrel - which may change in the future with increased competition from generic clopidogrel, ticagrelor, or other new antiplatelet agents. Lastly, while ticagrelor was not on the market at the time this study was initiated, it is available now and obviously needs to be part of the clinical decision.

It is also important to highlight that the perspective of this study was that of the thirdparty payer, and as a result, only direct medical costs were included in the analysis. If the study were conducted from a patient or societal perspective, then indirect costs, such as work lost productivity or earnings following a CV or non-CV event, would have been included. Inclusion of indirect costs would likely make the genotype-guided therapy option even more cost-effective 
because these costs are reduced when events are avoided, and while this would not change the results of the main analysis (where genotype-guided therapy was the best option), it might change the results of the analysis that includes generic clopidogrel, particularly considering that the WTP threshold for switching from the generic clopidogrel for all option to the genotypeguided therapy option was relatively low.

Despite these remaining questions, this is an important study in that it is one of the first to document that genotyped-guided pharmacotherapy can be both more clinically effective and less expensive than traditional therapy. This has important implications for pharmacists who in the future could play an integral role in prescribing decisions based on personal genetic information. Clinicians at Vanderbilt University recently announced efforts to genotype all potential clopidogrel candidates for CYP2C19 loss-of-function alleles. ${ }^{24}$ The genetic data are imbedded in the electronic medical record so that, in the event that a patient is prescribed clopidogrel, the genetic information is available for the clinician to consider. Other health care centers may follow suit. In fact, it is conceivable that patients may eventually be broadly genotyped a priori for multiple genotypes with implications for multiple drugs. This would essentially eliminate the cost of genotyping at the time of drug prescribing, since this cost would already be incurred, and eliminate the questions of when and who to genotype, further improving the cost effectiveness and utility of genotype-guided therapy. 


\section{References}

1. Brandt JT, Close SL, Iturria SJ, et al. Common polymorphisms of CYP2C19 and CYP2C9 affect the pharmacokinetic and pharmacodynamic response to clopidogrel but not prasugrel. J Thromb Haemost 2007;5:2429-2436.

2. Trenk D, Hochholzer W, Fromm MF, et al. Cytochrome P450 2C19 681G>A

polymorphism and high on-clopidogrel platelet reactivity associated with adverse 1-year clinical outcome of elective percutaneous coronary intervention with drug-eluting or bare-metal stents. J Am Coll Cardiol 2008;51:1925-1934.

3. Mega JL, Close SL, Wiviott SD, et al. Cytochrome p-450 polymorphisms and response to clopidogrel. N Engl J Med 2009;360:354-362.

4. Sibbing D, Koch W, Gebhard D, et al. Cytochrome 2C19*17 allelic variant, platelet aggregation, bleeding events, and stent thrombosis in clopidogrel-treated patients with coronary stent placement. Circulation 2010;121:512-518.

5. Collet JP, Hulot JS, Pena A, et al. Cytochrome P450 2C19 polymorphism in young patients treated with clopidogrel after myocardial infarction: a cohort study. Lancet 2009;373:309-317.

6. Shuldiner AR, O'Connell JR, Bliden KP, et al. Association of cytochrome P450 2C19 genotype with the antiplatelet effect and clinical efficacy of clopidogrel therapy. JAMA 2009;302:849-857.

7. Simon T, Verstuyft C, Mary-Krause M, et al. Genetic determinants of response to clopidogrel and cardiovascular events. N Engl J Med 2009;360:363-375.

8. Wiviott SD, Braunwald E, McCabe $\mathrm{CH}$, et al. Prasugrel versus clopidogrel in patients with acute coronary syndromes. N Engl J Med 2007;357:2001-2015. 
9. Walsh S. FDA Announces New Boxed Warning on Plavix

Alerts patients, health care professionals to potential for reduced effectiveness. 2010 [cited 2011 7/27/2011]; Available

from: http://www.fda.gov/NewsEvents/Newsroom/PressAnnouncements/ucm204253.htm

10. Wiviott SD, Trenk D, Frelinger AL, et al. Prasugrel compared with high loading- and maintenance-dose clopidogrel in patients with planned percutaneous coronary intervention: the Prasugrel in Comparison to Clopidogrel for Inhibition of Platelet Activation and AggregationThrombolysis in Myocardial Infarction 44 trial. Circulation 2007;116:2923-2932.

11. Gurbel PA, Bliden KP, Butler K, et al. Randomized double-blind assessment of the ONSET and OFFSET of the antiplatelet effects of ticagrelor versus clopidogrel in patients with stable coronary artery disease: the ONSET/OFFSET study. Circulation 2009;120:2577-2585.

12. Wallentin L, Becker RC, Budaj A, et al. Ticagrelor versus clopidogrel in patients with acute coronary syndromes. N Engl J Med 2009;361:1045-1057.

13. Mega JL, Close SL, Wiviott SD, et al. Cytochrome P450 genetic polymorphisms and the response to prasugrel: relationship to pharmacokinetic, pharmacodynamic, and clinical outcomes. Circulation 2009;119:2553-2560.

14. Wallentin L, James S, Storey RF, et al. Effect of CYP2C19 and ABCB1 single nucleotide polymorphisms on outcomes of treatment with ticagrelor versus clopidogrel for acute coronary syndromes: a genetic substudy of the PLATO trial. Lancet 2010;376:1320-1328.

15. Ticagrelor (Brilinta) - better than clopidogrel (Plavix)? Medical Letter 2011;53:69-71.

16. Scott SA, Sangkuhl K, Gardner EE, et al. Clinical Pharmacogenetics Implementation Consortium Guidelines for Cytochrome P450-2C19 (CYP2C19) Genotype and Clopidogrel Therapy. Clin Pharmacol Ther 2011;90:328-332. 
17. Reese E, Mullins CD, Beitelshees A. The cost-effectiveness of CYP2C19 genotype screening to select clopidogrel or prasugrel for antiplatelet therapy. Pharmacotherapy 2012. 18. Momary KM, Dorsch MP, Bates ER. Genetic causes of clopidogrel nonresponsiveness: which ones really count? Pharmacotherapy 2010;30:265-274.

19. Cayla G, Hulot JS, O'Connor SA, et al. Clinical, angiographic, and genetic factors associated with early coronary stent thrombosis. JAMA 2011;306:1765-1774.

20. Mega JL, Close SL, Wiviott SD, et al. Genetic variants in ABCB1 and CYP2C19 and cardiovascular outcomes after treatment with clopidogrel and prasugrel in the TRITON-TIMI 38 trial: a pharmacogenetic analysis. Lancet 2010;376:1312-1319.

21. Collet JP, Hulot JS, Anzaha G, et al. High doses of clopidogrel to overcome genetic resistance: the randomized crossover CLOVIS-2 (Clopidogrel and Response Variability Investigation Study 2). JACC Cardiovasc Interv 2011;4:392-402.

22. Sorich MJ, Vitry A, Ward MB, et al. Prasugrel vs. clopidogrel for cytochrome P450 2C19-genotyped subgroups: integration of the TRITON-TIMI 38 trial data. J Thromb Haemost 2010;8:1678-1684.

23. Scott SA, Sangkuhl K, Gardner EE, et al. Clinical Pharmacogenetics Implementation Consortium Guidelines for Cytochrome P450-2C19 (CYP2C19) Genotype and Clopidogrel Therapy. Clin Pharmacol Ther 2011;90:328-332.

24. Sue Hughes. Vanderbilt now also routinely gene testing for clopidogrel metabolizer status. theheart.org. [Clinical Conditions > Interventional/Surgery > Interventional/Surgery]; Oct 21, 2010. Accessed at http://www.theheart.org/article/1139495.do on Aug 11, 2011. 